\title{
Characterization and Expression of Two cDNA Encoding 3-Hydroxy-3-methylglutaryl coenzyme A Reductase Isoforms in Coffee (Coffea arabica L.)
}

\author{
Iris Tiski, ${ }^{1}$ Pierre Marraccini, ${ }^{2}$ David Pot, ${ }^{2}$ Luiz Gonzaga Esteves Vieira, ${ }^{3}$ and Luiz Filipe Protasio Pereira ${ }^{4}$
}

\begin{abstract}
In higher plants there are two independent pathways for isoprenoid biosynthesis, located in the cytosol (mevalonic acid or MVA pathway) or in the plastids [methylerythritol phosphate (MEP) pathway]. The 3-hydroxy3-methyglutaryl-CoA reductase (HMGR) is the first committed step in the MVA pathway. Using the information available from the Brazilian Coffee Genome Project, we found 13 ESTs that originated two isoforms, CaHMGR1 and CaHMGR2, for the enzyme HMGR of Coffea arabica. A complementary DNA encoding the isoform CaHMGR1 was cloned, and its complete nucleotide sequence determined. The full-length cDNA of CaHMGR1 was 2,242 bp containing a 1,812-bp ORF encoding 604 amino acids. Bioinformatic analyses revealed that the deduced CaHMGR1 had extensive homology with other plant HMGRs and contained two transmembrane domains and two putative HMGR binding sites and two NADP(H)-binding sites. Under normal growth conditions, transcripts of isoform CaHMRG1 were detected in fruit tissues (pulp, perisperm, and endosperm) only at the initial stages of development, flower buds and leaves. CaHMRG2 was expressed in all tissues and during all fruit development stages examined. These results suggest a constitutive expression of isoform CaHMGR2, while the isoform CaHMGR1 shows temporal and tissue-specific transcriptional activation.
\end{abstract}

\section{Introduction}

C OFFEE IS ONE OF THE MOST IMPORTANT world agricultural commodities. It is consumed for more than 800 million people (Illy, 2006). The genus Coffea contains about 100 species but commercial production is based only on species Coffea arabica (Arabica) and Coffea canephora (Robusta) that represent 70 and $30 \%$ of the total coffee market, respectively (Vieira et al., 2006). Although the cup quality is one of the main aspects for coffee consumption, little is known about several components that can contribute for quality (Leroy et al., 2006).

Arabica and Robusta species present differences in the contents of caffeine, trigonelline and chlorogenic acids as well as in the amount and composition of lipids. Lipids are important components of coffee beverage and aroma. Coffee oil is composed mainly of triacylglycerols. The relatively large unsaponifiable fraction is rich in diterpenes of the kaurane family, mainly cafestol $\left(\mathrm{C}_{20} \mathrm{H}_{28} \mathrm{O}_{3}\right)$ and kahweol $\left(\mathrm{C}_{20} \mathrm{H}_{26} \mathrm{O}_{3}\right)$, which have been receiving increasingly attention in recent years due to their physiological effects in human health (Speer and Kölling-Speer, 2006).
Cafestol and khaweol originated from the isoprenoid pathway. Isoprenoids are the most functionally and structurally varied group of plant metabolites. Isoprenoids are synthesized in all organisms but are especially abundant and diverse in plants, with more than 25,000 compounds reported to date in the plant kingdom (Croteau et al., 2000; Rodriguez-Conception 2010; Sacchettini and Poulter, 1997). Despite their diversity in functions and structures, all isoprenoids derive from the common-five carbon-building unit isopentenyl diphosphate (IPP) and its isomer dimethylallyl diphosphate (DMAPP) (Rodrigues-Conception and Boronat, 2002). In higher plants, two independent pathways located in separate intracellular compartments are involved in the biosynthesis of IPP and DMAPP (Fig. 1). In the cytosol, IPP is derived from the mevalonic acid (MVA) pathway (Chappell, 1995; Newman and Chappell, 1999; Qureshi and Porter, 1981), whereas in plastids, IPP is formed from pyruvate and glyceraldehyde 3-phosphate through the methylerythritol phosphate (MEP or nonmevalonate) pathway (Eisenreich et al., 1998; Rohmer, 1999). In the MVA pathway three molecules of acetyl-CoA condense successively to form 3-hydroxy-3-

\footnotetext{
${ }^{1}$ Biotechnology Graduation Program-UEL, Londrina, PR, Brazil.

${ }^{2}$ CIRAD, UMR AGAP Montpellier Cedex, France.

${ }^{3}$ IAPAR, CP 481, Londrina, PR, Brazil.

${ }^{4}$ EMBRAPA Café, PqEB, Brasília, DF, Brazil.
} 


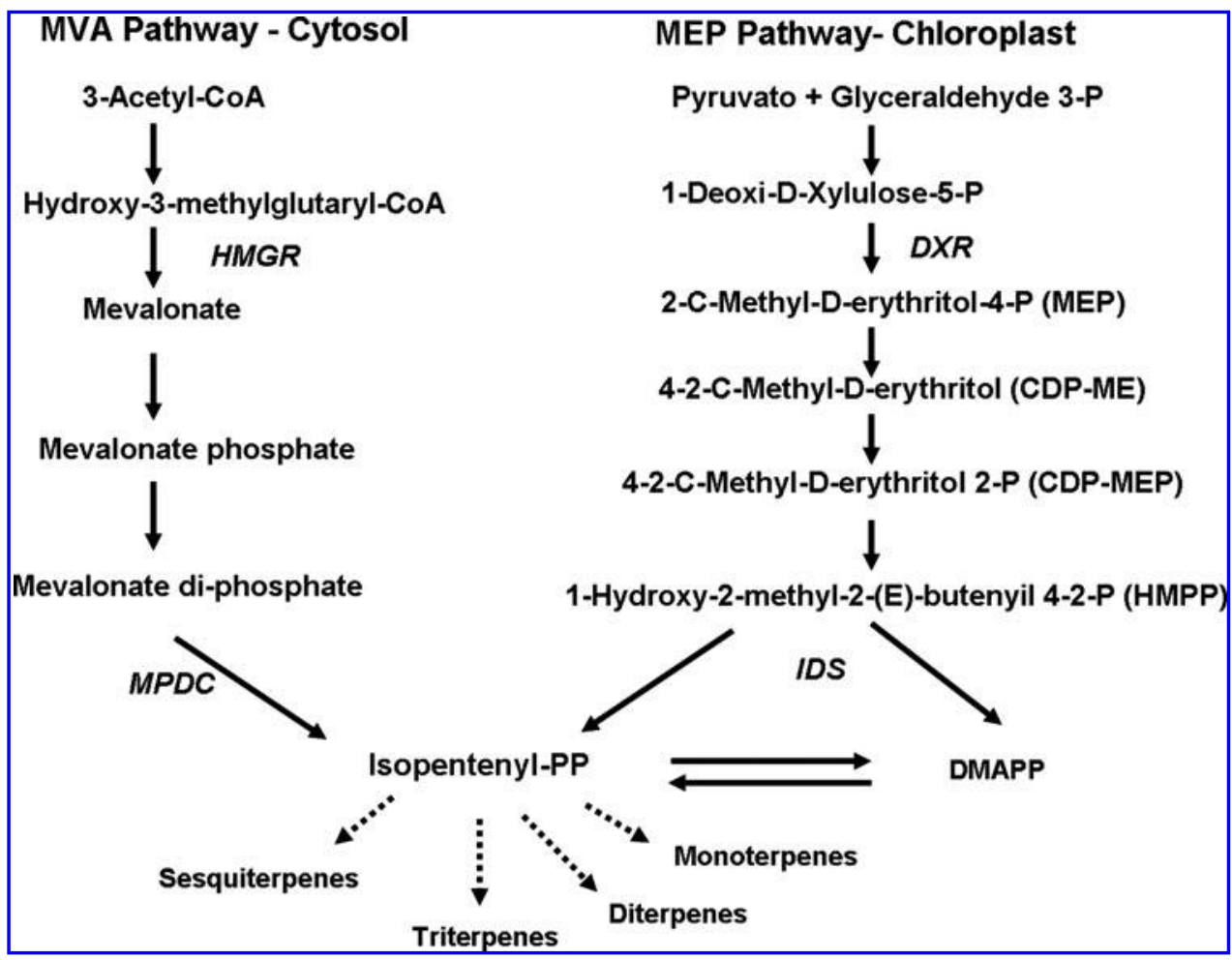

FIG. 1. Diagram of the isoprenoid pathway in the cytosol and in the plastids in plants.

methylglutaryl-CoA (HMG-CoA). This CoA derivative is reduced to mevalonate by HMG-CoA reductase (HMGR) (EC 1.1.1.34), the rate-limiting enzyme of the mevalonate pathway. Mevalonate is then phosphorylated twice and decarboxylated to form IPP, which is then converted to its isomer, DMAPP, by IPP isomerase. IPP and DMAPP synthesized in the mevalonate pathway are used as basic units in the biosynthesis of isoprenoids.

In higher plants, the gene HMGR is reported to be encoded by two or more isoforms. In Arabidopis thaliana, Cucumis melo, and Lycopersicum esculentum HMGR are encoded by two isoforms (Kato-Emori et al., 2001; Lange and Ghassemian, 2003; Narita and Gruissem, 1989), whereas in Solanum tuberosum and Hevea brasiliensis by three isoforms (Choi et al., 1992; Chye et al., 1992). These HMGR isoforms can be differentially expressed, depending on physiological conditions (Stermer et al., 1994; Weissenborn et al., 1995). Croteau et al. (2000) sugested that HMGR in plants is highly regulated and expressed in complex patterns, with individual genes exhibiting constitutive, tissue- or development-specific (Liao et al. 2009; Pateraki and Kanellis, 2010), hormones (Mansouri et al. 2009), or pathogen-inducible expression. An increase in HMGR transcription also has been reported after the application of salicylic acid or methyl jasmonate in Salvia miltiorrhiza and in xerophytes plants (Liao et al. 2009; Pateraki and Kanellis, 2010). Tissue wounding also stimulate an increase of HMGR transcription as well as other genes involved in the MVA and MEP pathway (Liu et al. 2010; Pateraki and Kanellis, 2010).

Herein, we report the characterization of a cDNA encoding a C. arabica HMG-CoA reductase. Also, the expression profile of two CaHMGR in various parts of the plant was investigated aiming to understand the process of formation of coffee isoprenoids.

\section{Material and Methods}

\section{Plant materials}

Fruits and tissues were harvested from 15-year-old plants of Coffea arabica cv. IAPAR 59 cultivated under field conditions at IAPAR (Instituto Agronômico do Paraná, Londrina, PR, Brazil). Fruits were collected every 4 weeks from flowering (September, 2005) up to complete maturation (May 2006). After collection, tissues were immediately frozen in liquid nitrogen and stored a $-80^{\circ} \mathrm{C}$ before being analyzed. Fruit tissues (perisperm, endosperm, and pulp) were separated and used independently to extract total RNA.

\section{In silico analysis}

A keyword search for HMGR ESTs of C. arabica was carried out on the database of the Brazilian Coffee Genome Project (http:/www.lge.lbi.unicamp.br/cafe/). This database contained 130,792 expressed sequence tag (ESTs) of C. arabica, 12,381 ESTs of C. canephora, and 10,566 ESTs of C. racemosa distributed into 37 cDNA libraries, sequenced from the $5^{\prime}$ end (Mondego et al., 2011). The sequences obtained were clusterized using the Sequencher v.4.1.4 software and BioEdit Sequence Alignment Editor v.7.0.8. For clusterization in the Sequencher the minimum match percentage and minimum overlap were 85 and 20\%, respectively. Contigs were analyzed using the basic local alignment search tool (BLAST) in the tBLASTx mode at NCBI database.

\section{Amplification of HMGRs}

To check if the assembled reads were part of the same HMGR contig or formed a unique contig different primer combinations were designed for the purpose of amplifying 
their open reading frames (ORFs) by RT-PCR. The primers designed according to conserved regions of related sequences were: (1) Fw-HMGRA 5'-GCC GGC GAA CAA CTC ATC AA-3' in the sense orientation, (2) Rv-HMGRB 5'-TTA CGG CCT CCA TCA TGG TG-3', and (3) Rv-HMGRC 5' - GCT GCA ATG GCT GAC ATC AGT-3' in the antisense orientations. PCR reactions $\left(25^{\prime} \mathrm{L}\right)$ were performed with $5 \mathrm{ng}$ cDNA of fruit tissues, $10 \mathrm{mM}$ TRIS-HCl pH8, 4; $50 \mathrm{mM} \mathrm{KCL}, 25 \mu \mathrm{M}$ dNTP, $2.5 \mathrm{mM} \mathrm{MgCl}_{2}, 1.0 \mathrm{U}$ de Taq polimerase (Invitrogen), and 10 pmol primer (sense and antisense). PCR conditions were: $2 \mathrm{~min}$ at $94^{\circ} \mathrm{C}$, followed by 40 cycles of $30 \mathrm{sec}$ at $94^{\circ} \mathrm{C}, 1 \mathrm{~min}$ at $57^{\circ} \mathrm{C}, 2 \mathrm{~min}$ at $72^{\circ} \mathrm{C}$, and a final cycle of $4 \mathrm{~min}$ at $72^{\circ} \mathrm{C}$ in a thermocycler PT- $100^{\mathrm{TM}}$ (MJ Research, Waltham, MA, USA). PCR products were visualized in $1 \%$ agarose gels and stained with ethidium bromide. PCR products were eluted from the gel and purified by ethanol precipitation in presence of $10 \%$ $(\mathrm{v} / \mathrm{v}) \mathrm{NaAc} \mathrm{pH} 5.2$ and ressuspended in water, either for sequencing or for producing probes for hybridizations.

\section{Sequencing and sequence analysis}

Clones containing sequences of CaHMGR1 were obtained from the clone collection of the Brazilian Coffee Genome Project. To obtain the complete sequence of CaHMGR1 of coffee, the clone containing the cDNA insert with the $5^{\prime}$ end region of the contig HMGRA was resequenced using the primers Fw-HMGRA (5'-GCC GGC GAA CAA CTC ATC AA- $\left.3^{\prime}\right)$ and Rv-HMGRB ( $5^{\prime}$-TTA CGG CCT CCA TCA TGG TG-3'). Sequencing was performed using DYEnamic ${ }^{\mathrm{TM}} \mathrm{ET}$ Terminator Cycle Sequencing Kit (GE HealthCare, Piscataway, NY, USA) in a MegaBace 1000 sequencer. The sequence obtained was analyzed using the basic local alignment search tool (BLAST) in the tBLASTx mode at NCBI database. The consensus sequence was translated using the ORF Finder available at NCBI and aligned with homologous protein sequences. The SGN Intron Finder tool (Mueller et al., 2005) program was used for predicting the presence of exons/ introns. The programs SignalP 3.0 and TMHMM2.0 (Emanuelsson et al., 2000) were used for predicting the presence of domains. Theoretical isoeletric point and molecular weight were calculated using ExPASy server (Gasteiger et al., 2003). The alignment with HMGR sequences of L. esculentum, N. tabaco, C. arabica, and C. melo was made using Vector NTI software (Invitrogen, Carlsbad, CA, USA), using standard parameters. Phylogenetic tree was constructed in Mega v.4.0 (Kumar et al., 2004) using UPGMA method. Bootstrap analysis was used to test the robustness of the UPMGA trees with 1000 replicates.

The nucleotide and deduced amino acid sequences of the full-length CaHMGR1 cDNA and partial fragment of CaHMGR2 gene described here have been submitted to GenBank and the assigned accession number are HQ540670 and HQ540671, respectively.

\section{Southern blot analysis}

For Southern blot analysis, $10 \mu \mathrm{g}$ of DNA (Chang et al., 1993, with modification) of leaves of Coffea arabica cv. IAPAR 59, C. eugenioides, and C. canephora var. Apoatã were digested with restriction endonuclease DraI, separated on a $0.8 \%(\mathrm{w} / \mathrm{v})$ agarose gel, transferred to nylon membranes and hybridized with an internal fragment of 1,474 bp of Coffea CaHMGR1 cDNA fragment labeled by random priming with $50 \mu \mathrm{Ci}$ of $\left[\alpha^{-}{ }^{32} \mathrm{P}\right] \mathrm{dCTP}$ according to Sambrook et al. (1989). Hybridization was performed overnight at $42^{\circ} \mathrm{C}$ using UltraHyb solution (Ambion, Austin, TX, USA) as previously described (Pereira et al., 2005). The membranes were washed as follows: two times SSC 1\%; SDS $0.1 \%$ for $20 \mathrm{~min}$ and SSC $1 \%$; SDS $0.1 \%$ for $20 \mathrm{~min}$ at $42^{\circ} \mathrm{C}$. The images were captured using fluorescent image analyzer FLA 3000 series (Fuji Photo Film Co., Lts. Tokyo, Japan).

\section{Analysis of expression by Northern blot}

For analysis of expression by Northern blot $10 \mu \mathrm{g}$ of total RNA was transferred to nylon membranes and hybridized with probes of CaHMGR1 and CaHMGR2 using UltraHyb solution as previously described for Southern blot. Total RNA was isolated (Chang et al., 1993, with modification) from different tissues of coffee like young flower buds $(1.5 \mathrm{~cm})$, mature flower $(2.0 \mathrm{~cm})$, expanded leaves $(10 \mathrm{~cm})$, young leaves $(4.0 \mathrm{~cm})$, branch, fruit, roots, pulp, perisperm, and endosperm from fruits of $C$. arabica $\mathrm{cv}$. IAPAR 59 at different stages of maturation.

\section{Results}

\section{In silico analysis of HMGR}

A search for HMGR ESTs conducted on the database of the Brazilian Coffee Genome Project resulted in 13 ESTs from C. arabica that exhibited of 43-90\% identity with HMGR gene of Arabidopsis thaliana (AY488113.1) at the nucleotide level. Assembly of all ESTs and alignment to Arabidopsis HMGR revealed three incomplete contigs that correspond to $5^{\prime}$ and $3^{\prime}$ regions of the Arabidopsis gene: contig A with 97-299 bp with $61 \%$ of identity ( $5^{\prime}$ end), contig B with $332-632$ bp and contig C with $447-632$ bp, with 83 and $89 \%$ of identity at the $3^{\prime}$ end (Fig. 2 ). The translated sequence of the contig A presented the highest similarity (73\%) with the N-terminal region of a consensus Nicotiana tabacum (AAB87727) HMGR protein, but

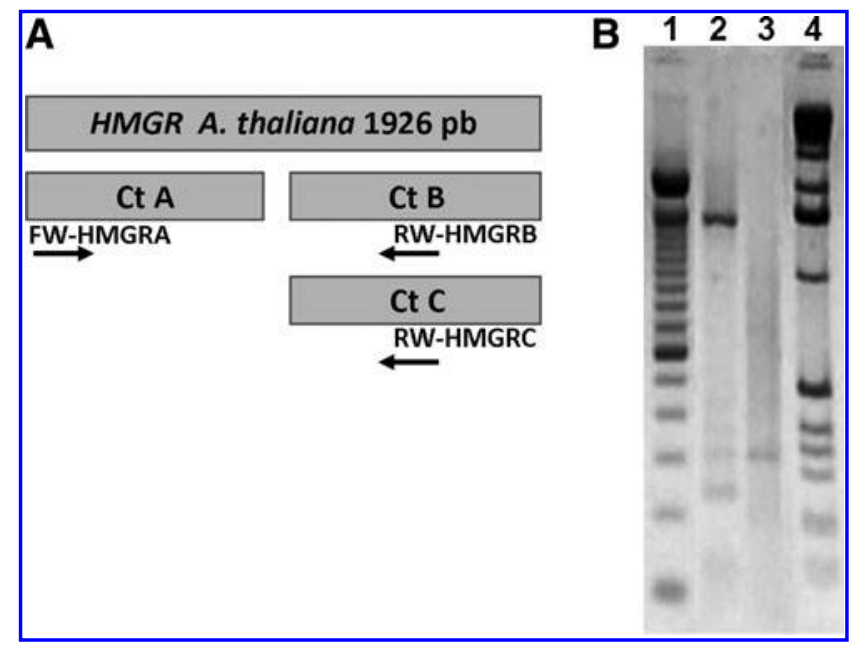

FIG. 2. Characterization of HMGR isoforms. (A) Contigs and their position in relation to a consensus $A$. thaliana sequence. (B) RT-PCR amplification of coffee HMGR. Columns: 1) Molecular weight 100 base pairs; 2) primers Fw-HMGRA and Rv-HMGRB; 3) Fw- HMGRA and Rv-HMGRC; 4) Molecular weight $1 \mathrm{~kb}$. 
with an incomplete C-terminal region. Contigs B and C also presented high similarity with the $\mathrm{C}$-terminal region of the same protein, but they are incomplete at $\mathrm{N}$-terminal region (Fig. 2A). The translated sequence of the contig $\mathrm{C}$ presented highest similarity ( $93 \%$ of identity) with the $\mathrm{C}$-terminal region of a consensus Morus alba (AAD03789) HMGR protein. The alignment of nucleotide sequences of contigs B and C in NCBI database showed e-value $1 \mathrm{e}^{-75}$ and identities of $75 \%$ between them. These results suggested that there are at least two genes encoding the HMGR enzyme in Coffea. Using only the in silico

\begin{tabular}{|c|c|}
\hline $\begin{array}{ll}\text { a } \\
\text { a. } \\
\text { v. } \\
\text { th }\end{array}$ & 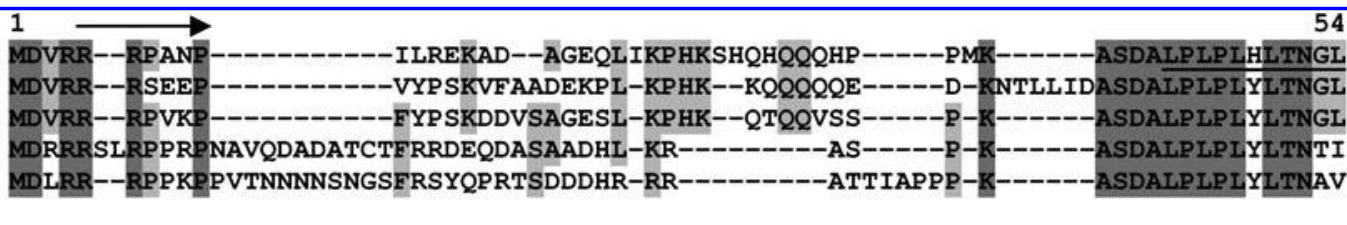 \\
\hline . & 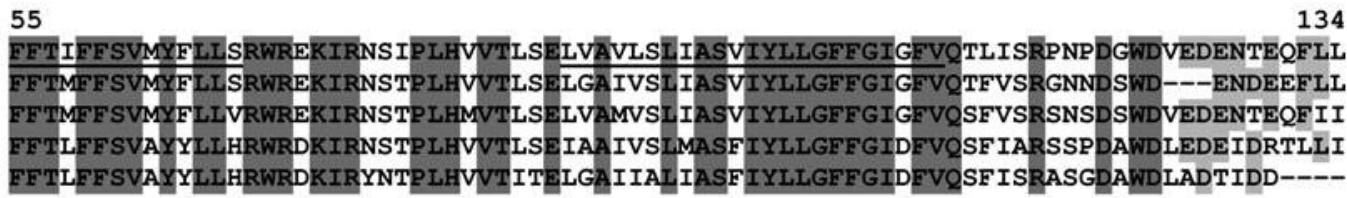 \\
\hline & 21 \\
\hline $\begin{array}{l}\text { C. arak } \\
\text { L. escu } \\
\text { N. tabc } \\
\text { c. melc }\end{array}$ & 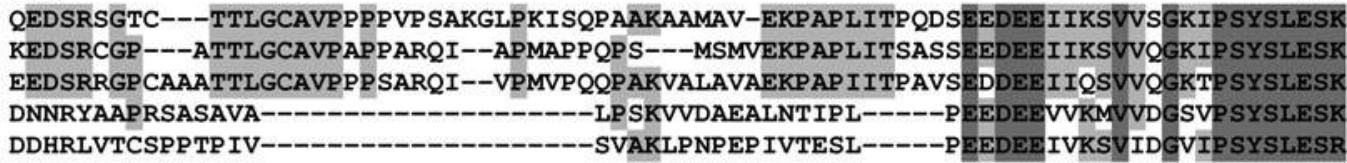 \\
\hline C. a & 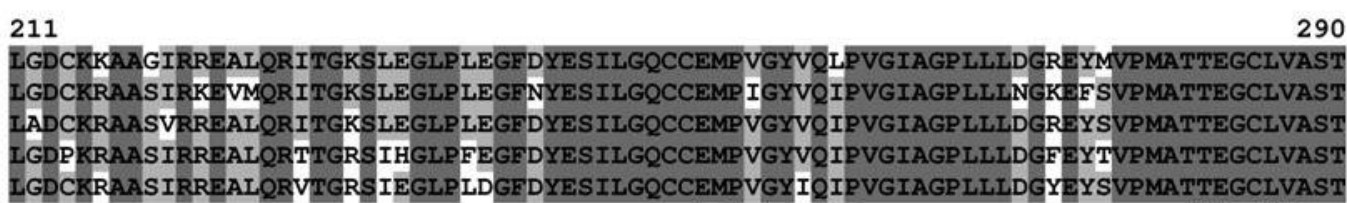 \\
\hline $\begin{array}{l}\text { C. } \\
\text { L. } \\
\text { N. } t \\
\text { C. } \\
\text { A. } \\
\text { a. }\end{array}$ & 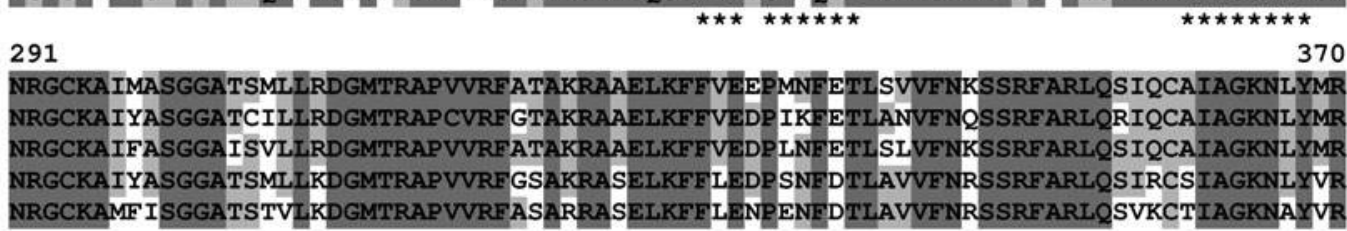 \\
\hline intum & 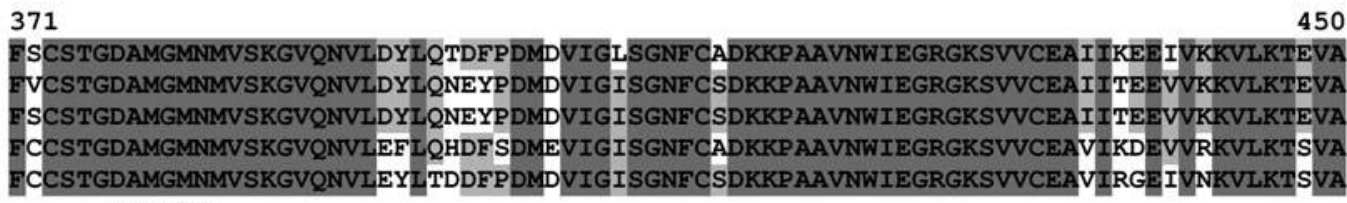 \\
\hline & \\
\hline $\begin{array}{l}\text { C. a } \\
\text { L. e } \\
\text { N. t } \\
\text { C. m }\end{array}$ & 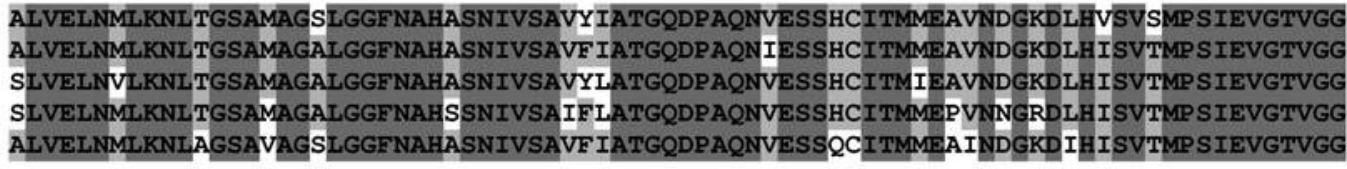 \\
\hline $\begin{array}{l}\text { C. arabica } \\
\text { L. esculentu } \\
\text { N. tabacum } \\
\text { C. melo } \\
\text { A. thaliana }\end{array}$ & $\begin{array}{l}531 \\
\text { GTKLASQSACLNLLRVKGANKFEAGANARLLATVVAGSVLAGELSLMSAISAGQLVSSH } \\
\text { GTQLASQSACLNLLGVKGANREAPGSNARLLATVVAGSVLAGELSLMSAISSGQLVNSH } \\
\text { GTQLASQSACLNLLGVKGANREAAGSNARLLATIVAGSVLAGELSLMSAISAGQLVKS } \\
\text { GTQLASQSACLNLLGVKGASKESP GANSRLLATIVAGSVLAGELSLMSAIAAGQLVRS } \\
\text { GTQLASQSACLNLLGVKGASTESPGMNARRLATIVAGAVLAGELSLMSAIAAGQLVRSH }\end{array}$ \\
\hline
\end{tabular}

FIG. 3. Multiple alignment of the protein sequence deduced from the Coffea arabica with HMGR sequences from Lycopersicon esculentum (AAB62581), Nicotiana tabacum (AAB87727), Cucumis melo (BAA36291), and Arabidopsis thaliana (P14891). Identical amino acids are indicated with black foreground and thick gray background. Conserved amino acids are denoted with black foreground and light gray background. Two putative transmembrane regions are indicated underlined (LPLHLTNGLFFTIFFSVMYFLLS and LVAVLSLIASVIYLLGFFGIGFV). Two putative HMG-CoA binding sites (EMPIGYVQIP and TTEGCLVA) are indicated with asterisks and two NADP(H)-binding sites (DAMGMNM and GTVGGGT) are indicated with black dots. Arrows indicated the position of primers Fw-HMGRA and Rv-HMGRB used to amplify the cDNA fragment. 
data, it was not possible to determine the complete cDNA sequence of Coffea HMGR isoforms.

\section{Characterization and sequencing of a full-length HMGR cDNA}

Primers were designed from highly conserved amino acid sequence of contigs $\mathrm{A}, \mathrm{B}$, and $\mathrm{C}$ to verify if the sequences of the three HMGRs contigs belong to the same or different isoform. When contig A forward primer (Fw-HMGRA) were used with the reverse primer of the contig $\mathrm{B}$ (Rv-HMGRB) for RT-PCR, it was possible to amplify a fragment about $1,560 \mathrm{bp}$, suggesting that they belong the same isoform, named CaHGMR1 (Fig. 2B). No fragment was amplified using the combination forward primer of the contig A (Fw-HMGRA) and the reverse primer of the contig $C$ (Rv-HMGRC), suggesting that the contig $\mathrm{C}$ is part of a different isoform, and thus called CaHMGR2. To close the gap between contigs A and B and obtain the complete sequence of CaHMGR1, the clone forming contig A with the cDNA insert, which has the longest 5'-UTR sequence, was resequenced using the primers Fw-HMGRA and Rv-HMGRB. The cDNA sequence of the CaHMGR1 comprises 2,242 bp, with the predicted presence of four exons and three introns (position 1186, 1368, 1715) with an ORF of $1,812 \mathrm{bp}$. The protein encoded by the CaHMGR1 cDNA has 604 amino acid residues and a predicted molecular mass of $64.7 \mathrm{kDa}$ and a deduced isoelectric point of 7.51.

The sequence analysis of the translated protein revealed that the functional motifs of CaHMGR1 were very similar to those of other plant HMGRs with two HMG-CoA binding motifs (EMPVGYVQLP and TTEGCLVA) and two NADPH binding motifs (DAMGMNM and GTVGGGT). The Signal p1.1 program showed a prediction signal anchor for sequence of the CaHMGR1. Two transmembrane regions were identified by TMHMM2.0 analysis: one was located between the amino acids 46 and 68, and the other was located between amino acid 88 and 110 along the polypeptide chain (Fig. 3). The alignment of the sequence of HMGR from $C$. arabica with the sequences of several representatives HMGRs from other plants shows that more differences were found in the $\mathrm{N}$-terminal region than in the C-terminal region (Fig. 3). The highest homology regions appeared around the substrate binding sites. Specifically, CaHMGR1 exhibits 77\% identity (84\% similarity) to HMGR2 of Solanum tuberosum and $83 \%$ identity ( $89 \%$ similarity) to HMGR of Nicotiana tabacum, $79 \%$ identity (85\% similarity) to HMGR2 Lycopersicum esculentum but only with $74 \%$ identity (85\% similarity) and $68 \%$ identity (79\% similarity) to Arabidopsis thaliana HMGR1 and HMGR2, respectively.

Phylogenetic analysis of HMGR enzymes from different species of plants using a yeast gene as outgroup revealed two distinctive groups: one group formed by the two monocotyledonous species (Oriza sativa and Zea mays), and other by dicots (Fig. 4). The deduced amino acid sequence of CaHMGR1 formed a clad with sequences from the Solanacea family.

\section{Southern blot analysis}

Southern Blot analysis using a CaHMGR1 probe was conducted to analyze the structure of the gene in Coffee spp. For the hybridization, the ORF region derived from cDNA was used as probe and the hybridization was carried out with high stringency washing. The hybridization showed a similar pattern of bands in C. eugenioides and C. canephora (three

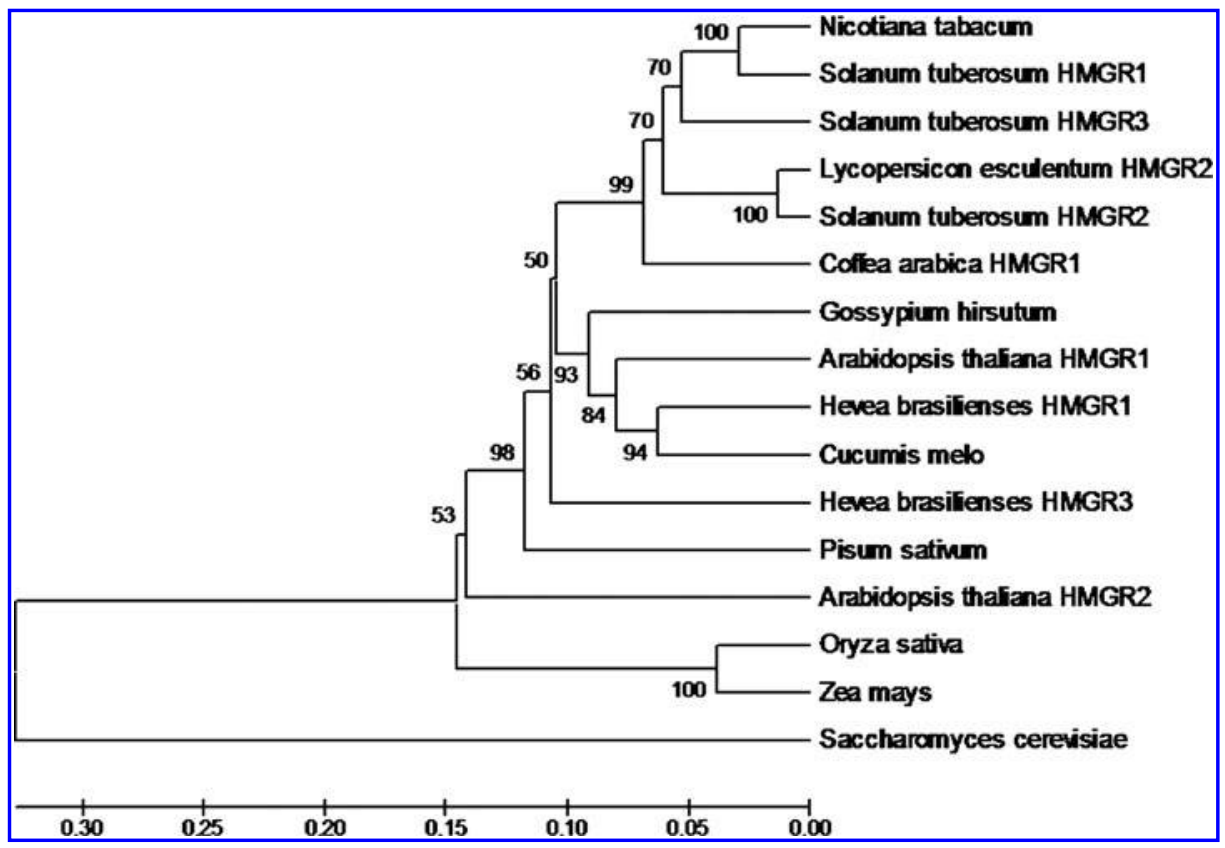

FIG. 4. Unrooted dendogram of HMGR sequences constructed by UPMGA method using Mega program. The sequences had been obtained through NCBI (National Center for Biotechnology Information http:/ / www.ncbi.nlm.nih.gov /): Nicotiana tabacum AAB87727; Solanum tuberosum HMGR1, P48020; Solanum tuberosum HMGR3, U51986; Lycopersicon esculentum AAB62581; Solanum tuberosum HMGR2, U51985; Gossypium hirsutum AAC05089; Arabidopsis thaliana HMGR1 P14891; Hevea brasiliensis HMGR1, X54659; Cucumis melo BAA36291; Hevea brasiliensis HMGR3, Q00583; Pisum sativum AAL37041; Arabidopsis thalianaHMGR2, L19262; Oryza sativum Q9XHL5; Zea mays O24594; Saccharomyces cerevisiae EDN64320. 


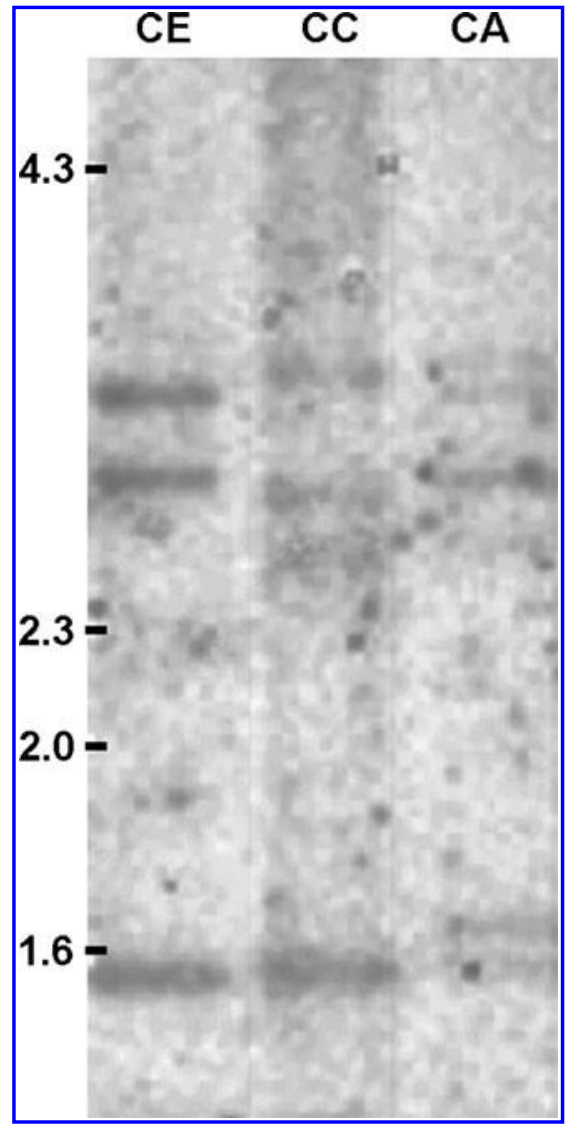

FIG. 5. Southern blot analysis of Coffea eugenioides (CE), Coffea canephora (CC), Coffea arabica (CA), genomic DNA hybridized with CaHMGR1 probes. Genomic DNA digested with DraI restriction enzymes were fractionated by electrophoresis on $0.8 \%(\mathrm{w} / \mathrm{v})$ agarose gel, and transferred to a nylon (Hybond- $\mathrm{N}^{+}$) membrane. Hybridizations were carried out with the probe CaHMGR1 corresponding to a $1,474 \mathrm{pb}$ internal fragment of CaHMGR1 cDNA. Molecular weight standards are indicated at the left in kilobases.

bands) (Fig. 5). In C. arabica a similar pattern was observed but with two more bands, probably due the tetraploid characteristic. These results suggest that CaHMGR1 belongs to a family of HMGR genes, with two or three copies for C. eugenioides and $C$. canephora and five or three copies for $C$. arabica. The probe CaHMGR1 did not show cross hybridization with CaHMGR2 probe (data not shown).

\section{Transcription levels of HMGRs}

Northern blots analysis with the CaHMGR1 probe detected transcripts in young flower buds, flower buds, and young leaves. It was very weak in expanded leaves and fruits, and it was not detected in roots and branches (Fig. 6). Cafestol and kahweol, originated from the isoprenoid pathway, are produced mainly in coffee fruits tissues. Transcritps of CaHMGR1 probe were verified in pulp, perisperm, and endosperm of coffee fruits during maturation. Although a weak detection of transcripts in whole fruits was observed, Northern blots analysis with the CaHMGR1 probe revealed transcripts during all maturation stages in pulp [55-251 days after flowering (DAF)], at the initial stage of development of perisperm (55

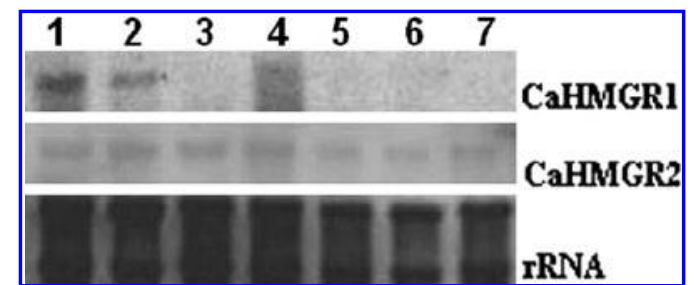

FIG. 6. Transcription pattern of CaHMGR1 and CaHMGR2. Total RNA (15 $\mu \mathrm{g})$ isolated from of (1) young flower buds; (2) mature flower buds; (3) expanded leaves; (4) young leaves; (5) branch; (6) fruit and (7) root of C. arabica. IAPAR 59 was separated in a formaldehyde-agarose gel and transferred onto a nylon membrane. Membranes hybridized with probes marked with dCTP $\alpha-32 \mathrm{P}$. Total RNA: gel of the samples for comparison of the used concentration.

DAF) and very weak signals in endosperm (83 DAF) (Fig. 7). Meanwhile, CaHMRG2 transcripts were observed in all samples analysed, in all different tissues of coffee fruits during maturation, as well as flower buds, leaves, branch, and roots (Figs. 6 and 7).

\section{Discussion}

Here we report that, through the application of bioinformatics analysis to the Coffee Genome Project EST database, we obtained the sequences of two 3-hydroxy-3methylglutaryl coenzyme A reductase (HMGR) isoforms. In addition, we identified a full-length clone encoding this enzyme and we reported the expression data in various coffee tissues. In Arabidopis thaliana, Cucumis melo, and Lycopersicum esculentum HMGR are encoded by two isoforms (KatoEmori et al., 2001; Lange and Ghassemian, 2003; Narita and Gruissem, 1989), in Solanum tuberosum and Hevea brasiliensis by three isoforms (Choi et al., 1992; Chye et al., 1992). The in silico analysis based on ESTs data associated with the PCR results indicated the expression of only two isoforms of HMGR in Coffea. Although it is posssible that another isoform is transcribed, the number of ESTs analysed (more than 153,000 for C. arabica plus 47,000 for C. canephora) strongly suggests that only two isoforms of HMGR may have an important role for isoprenoid biosynthesis via the mevalonate pathway in C. arabica. Wu et al. (2009), also identified only two HMGR isoforms after analyzing 6678 ESTs from flower, leaf, and root cDNA of American ginseng (Panax quinquefolius L.).

According to the in silico analyses, the deduced CaHMGR1 of coffee showed considerable identities with other known plant HMGRs proteins and contained two transmembrane domains, two HMGR binding motifs, and two NADPH binding motifs (Fig. 3) as in Cucumis melo (Kato-emori et al., 2001), Taxus media (Liao et al., 2004), and Corylus avellana (Wang et al., 2007). The protein encoded by the CaHMGR1 CDNA has a predicted molecular mass and a deduced isoelectric point that is very similar to the previously reported for other plants (Liao et al., 2004; Shen et al., 2006; Wang et al., 2007). The strongest amino acid homology was found to HMGR enzymes of N. tabacum, S. tuberosum, and L. esculentum. Phylogenetic relatedness of CaHMGR1 to known HMGRs from the Solanaceae family is illustrated in Figure 4. The tree shows the early split of monocots and dicots with a separate branch for yeast. The clad 


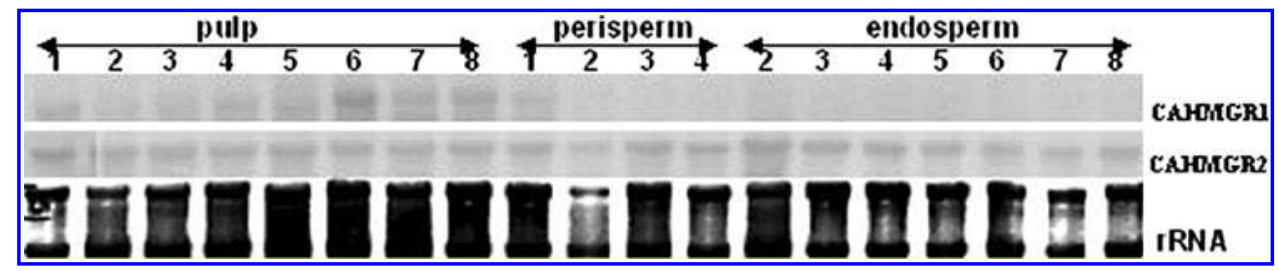

FIG. 7. Transcription pattern of CaHMGR1 and CAHMGR2. Total RNA (15 $\mu \mathrm{g})$ isolated from pulp; perisperm and endosperm of fruits of $C$. Arabica cv. IAPAR 59. Columns 1 to 11 correspond to months after flowering (AF) from the period of October (30 days AF) to May (240 days AF). Membranes hybridized with probes marked with dCTP ${ }^{\alpha} \mathrm{P}^{32}$. Total RNA: gel of the samples for comparison of the used concentration.

formed with CaHMGR1 and HMGR sequences of especies from the Solanaceae family corroborate with reports showing the proximity between Coffea from the Rubiaceae family with the Solanacea (Lin et al., 2005).

To investigate whether the CaHMGR1 gene belongs to a multigene family, genomic DNA of C. arabica and its parents, C. canephora and C. eugenioides were digested with DraI that had no restriction site within the probe region. For all three species, there were more than two distinct bands ranging from $4.3 \mathrm{~kb}$ to less than $1.6 \mathrm{~kb}$ (Fig. 4), which might be speculated that more than one sequence with homology to CaHMGR1 gene were present in Coffea genome, although there is also the possibility for the presence of restriction sites in the intron sequence of CaHMGR1. Venkatachalam et al. (2009) reported the presence of three introns in a rubber three HMGR gene. It was suggested that more than two copies of HMGR gene may exist in Solanum tuberosum and Hevea brasiliensis (Choi et al., 1992; Chye et al., 1992; Venkatachalam et al., 2009). So the possibility cannot be excluded that CaHMGR1 might be a two-copy gene, and the number of CaHMGR1 genes from C. arabica will be elucidated when more genomic information is available.

Low abundant transcripts of both CaHMGR1 and CaHMGR2 were detected by Northern blots. An additional confirmation of low HMGR transcription is that the sequence of the two isoforms of this gene were found only 13 times in a search of more than 200,000 ESTs from 37 cDNA libraries constructed by using mRNAs isolated from different tissues at different developmental stages. Analysis of coffee HMGR genes expression in all fruit tissues during the developmental stages studied revealed that the CaHMGR2 gene was expressed constitutively in all tissues examined but at very low levels (transcripts became visible only after $18 \mathrm{~h}$ exposure to the fluorescent image analyzer) (Figs. 6 and 7). Similar results were observed by Dudareva et al. (2005), who reported a constitutive and low gene expression level of a HMGR gene in leaves and floral tissues of snapdragon flowers (Antirrhinum majus). On the other hand, Northern blot results indicated abundant mRNAs encoding CaHMGR1 was only transcribed in floral buds and young leaves, whereas a weak signal was detected in whole fruits of $C$. arabica. The isoprenoids cafestol and kahweol are produced mainly in fruits of $C$. arabica (Speer and Kolling-Speer, 2001); however, fruits analysed in Northern blot are constituted of pulp and mainly endosperm (Geromel et al., 2006), which explain the weak detection of transcripts in whole fruits. Northern blot from developmental stages of fruit tissues detected CaHMGR1 transcripts in pulp, mainly at the initial stages of perisperm and in the endosperm. The transcription of CaHMGR1 for the perisperm tissue cor- relates to the observed amount of diterpenes kahweol and cafestol in this tissue reported by Dias et al. (2010), but surprisingly, did not show the amount of transcription expected for the endosperm. The overall low transcription level of both HMGR isoforms in coffee suggests that the activity of this enzyme may be subject to both posttranscriptional and posttranslational regulation. Dudareva et al. (2005) showed that HMGR is expressed constitutively in all tissues of Antirrhinum majus but at very low levels. Laule et al. (2003) and Nieto et al. (2009) showed the lack of correlation between gene expression patterns and the accumulation of isoprenoid metabolites in Arabidopsis, indicating that posttranslational processes may play an important role in regulating flux through isoprenoid metabolic pathways. The HMGR gene belongs to the MVA pathway, which is localized at the cytosol in opposition to the plastidic MEP pathway. Although the two pathways to isoprenoids (MAV and MEP) are compartmentalized, there is evidence that they cooperate in the formation of certain isoprenoids, probably as a consequence of metabolic crosstalk between the pathways via the plastid envelope membrane (Bick and Lange, 2003). These authors suggest the presence of an unidirectional proton symport system in plastid membranes for the export of specific isoprenoid intermediates between cytosolic and plastidial pathways. As the capacity of the MEP pathway to function or compensate the inhibition of MVA pathway is more pronounced than the opposite (Laule et al., 2003), it is tempting to speculate that the low gene expression level in coffee of both HMGRs, the key regulatory enzyme of the MVA pathway, indicates that the cytosolic pathway may not be the principal responsible for the production of isoprenoid precursors in coffee plants. Further experiments using RTqPCR for analysis of the transcription pattern of CaHMGR1 and CaHMGR2 from the MVA pathway, and DXR for the MEP pathway are being conducted in our lab.

To our knowledge, CaHMGR1 is the first reported gene involved in the MVA pathway from C. arabica. Further characterization of CaHMGR1 gene expression as well as other genes involved in the MVA and MEP pathways it will be very helpful to understand the importance of the cytosolic and plastidic pathways involved in the isoprenoid metabolism and their contribution for the terpenes biosynthesis. Furthermore, those genes can be potential targets for bioengineering terpenes in coffee beans.

\section{Conclusions}

The in silico and molecular analysis indicated the presence of only two isoforms of HMGR in Coffea arabica. For the 
deduced amino acid sequence of CaHMGR1, the strongest homology was found to HMGR enzymes of N. tabacum, S. tuberosum, and L. esculentum. Analysis of coffee HMGR genes expression revealed that the CaHMGR2 gene was expressed constitutively in all coffee tissues but at very low levels and CaHMGR1 was transcribed in floral buds, young leaves, in pulp. Transcription was detected in whole fruits and mainly at the initial stages of perisperm but at low levels in endosperm.

\section{Acknowledgments}

This project was supported by the Brazilian Consortium for Coffee Research (CPC) and Genocafé/FINEP. L.F.P. Pereira and L.G.E. Vieira received a research fellowship from CNPq. Iris Tiski received a graduate student fellowship from CPC.

\section{Author Disclosure Statement}

The authors declare that no conflicting financial interests exist.

\section{References}

Bick, J.A., and Lange, B.M. (2003). Metabolic cross talk between cytosolic and plastidial pathways of isoprenoid biosynthesis: unidirectional transport of intermediates across the chloroplast envelope membrane. Arch Biochem Biophys 415, 146154.

Chang, S., Puryear J., and Cairney, J. (1993). A simple and efficient method for isolating RNA from pine trees. Plant Mol Biol 11, 113-116.

Chappell, J. (1995). Biochemistry and molecular biology of the isoprenoid biosynthetic pathway in plants. Annu Rev Plant Physiol Plant Mol Biol 46, 521-547.

Choi, D., Ward, B.L., and Bostock, R.M. (1992). Differential induction and suppression of potato 3-hydroxy-3-methyglutaryl coenzymeA reductase genes in response to Phytophthora infestans and to its elicitor arachidonic acid. Plant Cell 4, 1333 1344.

Chye, M.L., Tan, C.T., and Chua, Nh. (1992). Three genes encode 3-hydroxy-3-methylglutaryl-coenzyme A reductase in Hevea brasiliensis: hmg1 and hmg3 are differentially expressed. Plant Mol Biol 19, 473-484.

Croteau, R., Kutchan, T.M., and Lewis, N.G. (2000). Natural products (secondary metabolites). In Biochemistry and Molecular Biology of Plants. B.B. Buchanan, W. Gruissem, and R.L. Jones, eds. (Americal Society of Plant Biology, Rockville, MD), pp. 1250-1268.

Dias, R.C.E., Campanha, F.G., Vieira, L.G.E., Ferreira, L.P., Pot, D., Marraccini, P., et al. (2010). Evaluation of kahweol and cafestol in coffee tissues and roasted coffee by a new highperformance liquid chromatography methodology. J Agric Food Chem 58, 88-93.

Dudareva, N., Andersson, S., Orlova, I., Gatto, N., Reichelt, M., Rhodes, D., et al. (2005). The nonmevalonate pathway supports both monoterpene and sesquiterpene formation in snapdragon flowers. Plant Biol 102, 933-938.

Emanuelsson, O., Nielsen, H., Brunak, S., and Von Heijne, G. (2000). Predicting subcellular localization of proteins based on their N-terminal amino acid sequence. I Mol Biol 300, 10051016.

Esenreich, W., Schwarz, M., Cartayrade, A., Arigoni, D., Zenk, M.H., and Backer, A. (1998). The deoxyxylulose phosphate pathway of terpenoid biosynthesis in plants and microorganisms. Chem Biol 5, 221-233.

Gasteiger, E., Gattiker, A., Hoogland, C., Ivanyi, I., Appel, R.D., and Bairoch, A. (2003). ExPASy: the proteomics server for indepth protein knowledge and analysis. Nucleic Acids Res. 31, 3784-3788.

Geromel, C., Ferreira, L.P., Guerreiro, S.M.C., Cavalari, A.A., Pot, D., Pereira, L.F.P., et al. (2006). Biochemical and genomic analysis of sucrose metabolism during coffee (Coffea arabica) fruit development. J Exp Bot 57, 3243-3258.

Illy, A. (2006). ASIC, 21st International Conference on Coffee Science, September 11-15, Montpellier.

Kato-Emori, S., Higashi, K., and Hosoya, K. (2001). Cloning and characterization of the gene encoding 3-hydroxy-3-methylglutaryl coenzyme A reductase in melon (Cucumis melo L. reticulatus). Mol Genet Genomics 265, 135-142.

Kumar, S., Tamura, K., and Nei, M. (2004). Mega 3.1 Integrated software for molecular evolutionary genetics analysis and sequence alignment. Brief Bioinfo 5, 150-163.

Lange, B.M., and Ghassemian, M. (2003). Genome organization in Arabidopsis thaliana: a survey for genes involved in isoprenoid and chlophyll metabolism. Plant Mol Biol 51, 925-948.

Laule, O., Furholz, A., Chang, H.S., Zhu, T., Wang, X., Heifetz, P.B., et al. (2003). Crosstalk between cytosolic and plastidial pathways of isoprenoid biosynthesis in Arabidopsis thaliana. Proc Natl Acad Sci USA 100, 6866-6871.

Leroy, T., Ribeyre, F., Bertrand, B., Charmetant, P., Dufour, M., Montagnon, C., et al. (2006). Genetics of coffee quality. Braz J Plant Physiol 18, 229-242.

Liao, P., Zhou, W., Zhang L., Wang, J., Yan X.M., Zhang, Y., et al. (2009). Molecular cloning, characterization and expression analysis of a new gene encoding 3-hydroxy-3-methylglutaryl coenzyme A reductase from Salvia miltiorrhiza. Acta Physiol Plant 31, 565-572.

Liao, Z., Tan, Q., Chai, Y., Zuo, K., Chen, M., Gong, Y., et al. (2004). Cloning and characterization of the gene encoding HMG-CoA reductase from Taxus media and its functional identification in yeast. Funct Plant Biol 31, 73-81.

Lin, C., Mueller, L.A., Mc Carthy, J., Crouzillat, D., Pétiard, V., Tanksley, S.D. (2005). Coffee and tomato share common gene repertoires as revealed by deep sequencing of seed and cherry transcripts. Theor Applied Genet 112, 114-130.

Liu, D., Zhang, L., Li, C., Yang, K., Wang, Y., Sun, X., et al. (2010). Effect of wounding on gene expression involved in artemisinin biosynthesis and artemisinin production in Artemisia annua. Russian J Plant Phys 57, 882-886.

Mansouri, H., Asrar, Z., and Mehrabani, M. (2009). Effects of gibberellic acid on primary terpenoids and 9-tetrahydrocannabinol in Cannabis sativa at flowering stage. I Integrative Plant Biol 51, 553-561.

Mondego, J., Vidal, R., Carazzolle, M.F., Tokuda, E.K., Parizzi, L.P., Costa, G.G.L., et al. (2011) An EST-based analysis identifies new genes and reveals distinctive gene expression features of Coffea arabica and Coffea canephora. BMC Plant Biol 11, 30.

Mueller, L.A., Solow, T.H., Taylor, N., Skwarecki, B., Buels, R., Binns, J., et al. (2005). The SOL Genomics Network. A comparative resource for solanaceae biology and beyond. Plant Physiol 138, 1310-1317.

Newman, J.D., and Chappell, J. (1999). Isoprenoid biosynthesis in plants: carbon partitioning within the cytoplasmic pathway. Crit Rev Biochem Mol Biol 34, 95-106.

Narita, J.O., and Gruissem, W. (1989). Tomato hydroxymethylglutaryl-CoA reductase is required early in fruit development but not during ripening. Plant Cell 1, 181-190. 
Nieto, B., Forés, O, Arró, M., and Ferrer, A. (2009). Arabidopsis 3-hydroxy-3-methylglutaryl-CoA reductase is regulated at the post-translational level in response to alterations of the sphingolipid and the sterol biosynthetic pathways. Phytochem 70, 53-59.

Pateraki, I., and Kanellis, A.K. (2010). Stress and developmental responses of terpenoid biosynthetic genes in Cistus creticus subsp. creticus. Plant Cell Rep 29, 629-641.

Pereira, L.F.P., Galvão, R.M., Kobayashi, A.K., Cação, S.M.B., and Vieira, L.G.E. (2005). Ethylene production and acc oxidase gene expression during fruit ripening of Coffea arabica L. Braz J Plant Physiol 17, 283-289.

Qureshi, N., and Porter, W. (1981). Conversion of acetyl-coenzyme A to isopentenyl pyrophosphate. In Biosynthesis of Isoprenoids Compounds. J.W. Porter and S.L. Spurgcon, eds. (Wiley, New York), pp. 47-94.

Rodriguez-Conception, M. (2010). Supply of precursos for carotenoid biosynthesis in plants. Arch Biochem Biophysics 504, $118-122$.

Rodriguez-Conception, M., and Boronat, A. (2002). Elucidation of the methylerythritol phosphate pathway for isoprenoid biosynthesis in bacteria and plastids. A metabolic milestone achieved through genomics. Plant Physiol 130, 1079-1089.

Rohmer, M. (1999). A mevalonate-independent route to isopentenyl diphosphate. In Comprehensive Natural Product Chemistry. D.E. Cane, ed. (Pergamon, Oxford), pp. 45-68.

Sacchettini, J.C., and Poulter, C.D. (1997). Creating isoprenoid diversity. Science 277, 1788-1789.

Sambrook, J., Fritsch, E.F., and Maniatis, T. (1989). Molecular Cloning: A Laboratory Manual (Cold Spring Harbor Laboratory Press, Cold Spring Harbor, NY).

Shen, G.A., Pang, Y.Z., Wu, W.S., Liao, Z.H., Zhao, L.X., Sun, X.F., et al. (2006). Cloning and characterization of a rootspecific expressing gene encoding 3-hydroxy-3-methylglutaryl coenzyme a reductase from Ginkgo biloba. Mol Biol Rep 33, 117-127.

Speer, K., and Kolling-Speer, I. (2001). Lipids. In Coffee: Recent Developments. R.J. Clarke and O.G. Vitzthum, eds. (Blackwell Science, New York), pp. 33-49.
Speer, K., and Kölling-Speer, I. (2006). The lipid fraction of the coffee bean. Braz J Plant Physiol 18, 201-216.

Stermer, B.A., Biancehini, G.M., and Korth, K. (1994). Regulation of HMG-CoA reductase activity implants. J Lipid Res 35, 1133-1140.

Venkatachalam, P., Priya, P., Jayashree, R., Rekha, K., and Thulaseedharan, A. (2009). Molecular cloning and characterization of a 3-hydroxy-3-methylglutaryl-coenzyme A reductase 1 (hmgr1) gene from rubber tree (Hevea brasiliensis Muell. Arg.): a key gene involved in isoprenoid biosynthesis. Physiol Mol Biol Plants 15, 133-142.

Vieira, L.G.E., Andrade, A.C., Colombo, C.A., Heloneida de Araújo Moraes, A., Metha, A., Carvalho de Oliveira, A., et al. (2006). Brazilian coffee genome project: an EST-based genomic resource. Braz J Plant Physiol 18, 95-108.

Wang, Y., Guo, B., Zhang, F., Yao, H., Miao, Z., and Tang, K. (2007). Molecular cloning and functional analysis of the gene encoding 3-hydroxy-3-methylglutaryl coenzyme A reductase from hazel (Corylus avellana L. Gasaway). J Biochem Mol Biol 40, 861-869.

Weissenborn, D.L., Denbow, C.J., Laine, M., Lang, S.S., Yang, Z., $\mathrm{Yu}, \mathrm{X}$., et al. (1995). HMG-CoA reductase and terpenoid phytoalexins: molecular specialization within a complex pathway. Physiol Plant 93, 393-400.

Wu, Q., Song, J., Sun, Y., Suo, F., Li, C., Luo, H., et al. (2010). Transcript profiles of Panax quinquefolius from flower, leaf and root bring new insights into genes related to ginsenosides biosynthesis and transcriptional regulation. Physiol Plant 138, 134-149.
Address correspondence to: Luiz Filipe Protasio Pereira EMBRAPA Café PqEB, Ed Sede, AV. W3 N (Final) 70770-901 Brasília, DF, Brazil

E-mail: filipe.pereira@embrapa.br 
